\title{
Age and the Egocentric Constraint on Coordination Stability:
}

\author{
An Exploratory Report \\ Andrew D Wilson, ${ }^{1^{*}}$ \\ Shaochen Huang ${ }^{2}$, \\ Geoffrey P. Bingham ${ }^{3}$,
}

\&

Qin Zhu

${ }^{1}$ Psychology, School of Social Sciences, Leeds Beckett University, Leeds, UK
${ }^{2}$ Kinesiology and Health, University of Wyoming, Laramie, WY, USA
${ }^{3}$ Psychological and Brain Sciences, Indiana University, Bloomington, IN, USA

* Corresponding Author

Email: a.d.wilson@leedsbeckett.ac.uk/ DrAndrewDWilson@gmail.com

Web: https://cognitioninaction.wordpress.com/

Twitter: @PsychScientists

All data and a preprint available at https://osf.io/9qtk5/ 


\section{Abstract}

The stability of coordinated rhythmic movements is primarily affected by the target relative phase. Relative phase can be identified in each of two frames of reference (an external, allocentric frame and a body-centred, egocentric frame) and both constrain stability. In the allocentric frame, coordinations that involve isodirectional movement $\left(0^{\circ}\right.$ mean relative phase) are more stable than those that do not. In the egocentric frame, coordinations that involve simultaneous use of homologous muscles (in-phase) are more stable than those that do not. The origin of the allocentric constraint is the visual perception of relative phase. The origin of this egocentric frame of reference is still unclear, although it is typically discussed in terms of neural crosstalk. Pickavance, Azmoodeh \& Wilson (2018) proposed that the egocentric constraint is also perceptual, based in the haptic perception of relative phase. As an initial step in pursuing this hypothesis, this exploratory report examines some data from two recent studies on the effect of ageing on performing and learning coordinated rhythmic movements. We show that participants in their 20 s show a strong egocentric effect in their coordination production, while this disappears in participants in their 60s. Participants in their 50s show an intermediate effect. We propose that a perceptual hypothesis is the best explanation of this age-related change, and lay out how to pursue hypothesis-driven tests in the future. 


\section{Introduction}

Coordinated rhythmic movement is a commonly used laboratory task for researching the acquisition and performance of skilled actions. In this task, participants are asked to produce oscillating movements of a limb (e.g. a finger, an arm, or a leg) and coordinate those movements with oscillating movements of either another limb (theirs or someone else's), or a computer display. The measure of coordination between such movements is relative phase (the relative position of the two oscillators within their respective cycles) and the stability of coordinated rhythmic movements is primarily a function of the target relative phase. The basic pattern is that $0^{\circ}$, in-phase coordinations are the most stable, $180^{\circ}$, anti-phase coordinations are stable but less so, and intermediate coordinations cannot be maintained without training (see Kelso, 1995 for a more detailed overview). Why does coordination stability vary with relative phase? The actions are not complex and can be produced simultaneously with ease if there is no requirement to coordinate them. This means that there is something about the coordination requirement that produces these effects, and so research tries to answer the question 'what is relative phase, from the point of view of the person?'

There are two answers (Swinnen, 2002). Assuming the movements are along parallel paths in 3space, the first identifies relative phase in terms of the relative direction of movement of the oscillators in an external, allocentric frame of reference. When the oscillators are moving in the same direction at the same time, they are at $0^{\circ}$ mean relative phase; when they are moving in the opposite direction at the same time, they are at $180^{\circ}$ mean relative phase. Everything in between requires practice to produce. There is extensive research supporting the hypothesis that people can perceive relative phase in terms of the allocentric relative direction of motion (e.g. Bingham, Schmidt \& Zaal, 1999; Bingham, Zaal, Shull \& Collins, 2000; Brandes, Rezvani and Heeds (2017); Mechsner, Kerzel, Knoblich \& Prinz, (2001); Snapp-Childs, Wilson \& Bingham, 2011; Swinnen, 2002; Wilson \& Bingham, 2008; Wilson, Collins \& Bingham 2005a, b; Zaal, Bingham \& Schmidt, 2000). 
The second answer identifies relative phase with respect to the body, which defines an internal, egocentric frame of reference. When the oscillators are moved symmetrically about the body's sagittal midline (e.g. in-out/in-out movements) they are in-phase and stable; when they are moved asymmetrically relative to that midline (e.g. left-right/left-right) they are in anti-phase and are less stable. Again, intermediate timing relations require practice to produce. There is also extensive research supporting the hypothesis that this egocentric constraint shapes coordinated rhythmic movement stability (see Swinnen, 2002 for a review). In fact, when the egocentric and allocentric constraints are pitted against one another experimentally, the egocentric constraint tends to dominate, with larger effect sizes (e.g. Kagerer, Summers, \& Semjen, 2003; Li, Levin, Forner-Cordero, Ronsse \& Swinnen, 2008; Peper, de Boer, de Poel, \& Beek, 2008; Temprado, Swinnen, Carson, Tourment \& Laurent, 2003). However, see Brandes et al (2017) and Mechsner et al (2001) for evidence that the allocentric constraint can dominate.

\section{What Implements the Egocentric Constraint?}

The allocentric constraint is implemented by the relative motion of the oscillators being projected into the optic array and that optic flow being detected and used as information for relative phase. The optic array is measured by vision in its allocentric frame of reference, and therefore relative phase measured in the optic array is in that allocentric frame. The question remains, however - how exactly is the egocentric constraint being implemented? What causes the non-visual contribution to coordination stability to occur within an egocentric frame of reference?

The main hypothesis about the egocentric constraint is that it arises from neural cross-talk during the planning and execution of limb movements (e.g. Beek, Peper \& Daffertshofer, 2002; Cattaert, Semjen \& Summers, 1999; Daffertshofer, Peper, \& Beek, 2005; Marteniuk, MacKenzie \& Baba, 1984; Swinnen, Young, Walter \& Serrien, 1991). Cross-talk occurs when the motor commands to one limbs also leak over into the other limb. Egocentric in-phase movements requires the simultaneous use of homologous muscle groups. This makes the motor commands from the two hemispheres essentially 
the same in content and timing, minimising interference from any cross-talk and allowing the resulting coordination to remain stable. That interference increases as the timing relation between the limbs moves towards egocentric anti-phase, due to the need for simultaneous use of nonhomologous muscle groups. Motor commands are presumed to occur within an egocentric frame of reference, and therefore the effect of muscle homology/neural cross-talk also occurs within an egocentric frame.

However, there is evidence that the egocentric frame is not being caused by muscle homology or patterns of muscle activation. Swinnen, Dounskaia, Levin \& Duysens (2001) and Swinnen, Dounskaia \& Duysens (2002) experimentally manipulated muscle homology and activation requirements in coordinated rhythmic movements and found that the egocentric constraint persisted independently of those requirements. They concluded that "the present findings provide converging evidence for the existence of an egocentric reference frame that goes beyond muscle homology" (Swinnen et al, 2002, pg 468). Limb direction is a primary parameter in the cortex, implemented with population coding (e.g. Georgopoulos, 1991, 1995) and this neural encoding occurs in an egocentric frame of reference. The egocentric constraint is still coming from the brain, but via a different proposed mechanism (limb direction rather than crosstalk).

The fact that the egocentric constraint is also about relative direction of oscillator motion, rather than the behaviour of muscles, also raises the possibility that this constraint could also be caused by the haptic perception of coordination via relative direction. From this (more ecological) point of view, the brain is not imposing the egocentric frame of reference. Rather, this is the frame of reference in which haptic perception of coordination occurs. Research has demonstrated that haptic perception can occur in both an egocentric and allocentric frame (Kappers, 2005, 2007) and so this hypothesis, while tentative, is initially plausible. It also provides a solely information based way of explaining the coordination effects, rather than needing a mix of informational and causal theories of perception; it has parsimony. 
Pickavance, Azmoodeh \& Wilson (2018) described how this reframing can be tested using the same tools we have used in the past to test the allocentric perception of relative phase (e.g. Bingham et al, 1999, 2000; Snapp-Childs et al, 2011; Wilson \& Bingham, 2008; Wilson et al, 2005a, b; Zaal et al, 2000). The current paper is an initial, exploratory examination of this hypothesis using some existing data sets. The goal of this paper is to identify patterns in data that can be related to perceptual mechanisms and that can help generate specific hypotheses for future work targeting the perceptual hypothesis.

\section{Age Related Changes in the Perception of Relative Phase}

We have recently published a series of papers looking at how age affects the acquisition of novel coordinations, specifically $90^{\circ}$ mean relative phase (Coats, Snapp-Childs, Wilson \& Bingham, 2012; Coats, Wilson, Snapp-Childs, Fath \& Bingham, 2014; Ren, Huang, Zhang, Zhu, Wilson, Snapp-Childs \& Bingham, 2015; Zhu, Mirich, Huang, Snapp-Childs, \& Bingham, 2017). What we have found is a precipitous drop in learning rates occurring in the 50s (the '50s cliff'; Coats et al, 2014), and we have recently shown that this is not due to known age-related changes in the visual detection of motion information - a haptically trained group also showed the cliff (Ren et al, 2015; Zhu et al, 2017). At the time, we interpreted this as showing the age-related changes were not caused by changes to visual perception, but by changes to perceptuo-motor learning ability more generally. However, an alternative explanation is that the deficit is still perceptual, and that the haptic perception of relative phase changes with age in similar ways to the visual perception of relative phase.

To explore the relationship between visual and haptic specification of relative phase more closely, Zhu et al (2017) trained three age-groups (20s, 50s and 60s) on a $90^{\circ}$ coordination using either haptic or haptic+visual feedback. They replicated the basic effect of age on learning performance. In addition, they found that within age group, both feedback groups learned equally well; however, the haptic+visual group's improvement did not transfer to a haptics-only test. The first group learned to perceive relative phase visually, which did not enable them to also perceive relative phase haptically. 
The authors concluded that the information for relative phase is modal, which would be expected if the two perceptual systems work in different frames of reference. A more recent follow-up (Bingham, Snapp-Childs and Zhu, submitted) has pursued this and confirmed that learning is modality specific.

\section{The Current Study}

None of the above studies were specifically designed to contrast the allocentric and egocentric constraints on coordination stability. However, both Ren et al (2015) and Zhu et al (2017) used bimanual tasks using side-to-side motions, and both assessed the performance at $0^{\circ}$ and $180^{\circ}$ at Baseline, Post Training and Retention. In these tasks, $0^{\circ}$ was defined allocentrically as isodirectional motion of the coordination feedback display (Wilson, Snapp-Childs, Coats \& Bingham, 2010) and was produced using side-to-side movements of the occluded arms. These actions are egocentrically antiphase movements. $180^{\circ}$ was the opposite; non-isodirectional allocentric motion of the feedback display was produced via egocentrically in-phase movements. The two frames of reference are in competition, and we can examine which dominates and whether that changes with age.

We are therefore using this existing data in an exploratory manner as an initial foray into the haptic perception of relative phase hypothesis. Our goal is to identify what happened in the relevant conditions of the two studies, and to identify whether that pattern changed with age. We have no specific hypotheses at this point; instead, we wish to use this data to generate hypotheses for future research into this perceptual account of the egocentric constraint. 


\section{Method}

We will be examining data from the Baseline assessment sessions performed in Ren et al (2015) and Zhu et al (2017). Refer to these papers for the full details and goals of those studies, but we will now summarise the details relevant for the current analysis.

\section{Participants}

Ren et al (2015) reported data for participants aged in their 20s, 50s, 60s and 70s ( $n=12$ per group). There were two identical Assessment sessions, at Baseline and Post-Training, in which performance at $0^{\circ}$ (anti-phase), $180^{\circ}$ (in-phase) and $90^{\circ}$ (out of phase) was tested. Training then entailed learning to move at $90^{\circ}$ with either haptic or visual feedback.

Zhu et al (2017) reported data for participants aged in their 20s and 50s ( $n=10$ per group). They also collected but did not report an incomplete dataset from people in their $60 \mathrm{~s}$ ( $\mathrm{n}=8$ for the haptic training group, $\mathrm{n}=3$ for the visual training group). There was also another data set available from this group, testing Age $=20 s$ ( $n=6$ for the haptic training group, $n=5$ for the visual training group). We will use this additional Assessment data in our analysis for some additional power. There were three identical Assessment sessions, at Baseline, Post-Training and Retention, in which performance at $0^{\circ}$ (anti-phase), $180^{\circ}$ (in-phase) and $90^{\circ}$ (out of phase) was tested. Training was again about learning $90^{\circ}$ with either haptic or visual feedback, this time with twice the amount of training as Ren et al (2015).

The combined sample sizes were therefore

- 20s: Visual Training $n=27$, Haptic Training $n=28$

- 50s: Visual Training $n=22$, Haptic Training $n=22$

- 60s: Visual Training $n=15$, Haptic Training $n=20$ 


\section{Procedure}

In the Baseline assessment sessions, the two training Groups saw different Visual Feedback displays. The Visual Training group were shown a visual demo of the target relative phase, presented allocentrically using a coordination display (Wilson et al, 2010). $0^{\circ}$ is when the two dots on the display oscillate from side to side moving in the same direction at the same time. To produce this $0^{\circ}$ behaviour, participants had to move two joysticks side to side doing the same thing at the same time; egocentrically, this is anti-phase coordination. $180^{\circ}$ was the reverse; allocentrically, the dots moved in the opposite direction at the same time, and to produce this, people moved the joysticks out and in at the same time (egocentrically this is in-phase coordination). The two constraints were therefore both present and in direct opposition. When the person was moving at the target relative phase $+/-20^{\circ}$, the dots changed colour from white to green (coordination feedback; Wilson et al, 2010). We shall refer to this group as the Coordination Display group.

The Haptic Training group were provided with a haptic demo of the target relative phase, in which a coach moved the joysticks while the person rested their hands on them. Relative phase was again defined allocentrically, so " 0 " and " $180^{\circ}$ " in this group involved the same movements as in the Coordination group (i.e. anti-phase and in-phase, respectively). The visual feedback display showed a single static dot, and this dot changed colour to green when the person was moving at the target relative phase $+/-20^{\circ}$. This meant that there was no visual, allocentric, motion information about the person's relative phase, just a hot/cold signal about accuracy. Only the egocentric constraint was able to affect coordination stability. We shall refer to this group as the Static Display group.

\section{Analysis Design}

Because the Baseline sessions were identical and comparable (no confound of training) we have combined the data from the three age groups the two studies had in common and analysed them in a three factor mixed analysis of variance (ANOVA). There was one within-subjects factor, Relative Phase, with two levels ( $0^{\circ}$ (Anti-Phase), $180^{\circ}$ (In-Phase)). There were two between subject factors. 
The first was Age, with three levels (20s, 50s and 60s) and the second was Allocentric Feedback, with 2 levels (Coordination, Static). The dependent variable was the proportion of the trial spent moving at the target relative phase, $+/-20^{\circ}$ (Proportion-Time-on-Target, PTT20). This is a valid and effective measure of coordination stability that has been used extensively in learning studies (Snapp-Childs et al, 2011, 2015).

\section{Results}

Refer to Figure 1. There was a significant main effect of Relative Phase, $F(1,128)=8.7, p<.01$, and a significant main effect of Age, $F(2,128)=5.4, p<.01)$, but these were both superseded by a significant Relative Phase ${ }^{*}$ Age interaction, $F(2,128)=3.7, p<.05$.

On the basis of the ANOVA and the data in Figure 1, we probed this interaction with 4 post-hoc, oneway, paired-sample t-tests of the hypothesis that coordination stability at $0^{\circ}$ (Anti-Phase) was lower than at $180^{\circ}$ (In-Phase). We compared stability data for the 20 s and 50 s groups from the Coordination and Static conditions separately. We did not test the 60 s data as the means were virtually identical, to minimise the number of post-hoc tests. The Bonferroni-corrected significance value for each t-test was therefore $p<.0125$.

For the Age $=20 \mathrm{~s}$ participants, $180^{\circ}$ (In-Phase) was significantly more stable than $0^{\circ}$ (Anti-Phase) in both the Coordination condition $(t(26)=-2.9, \mathrm{p}<.001)$ and the Static condition $(\mathrm{t}(27)=-3.2, \mathrm{p}<.01)$. For the Age $=50$ s participants, the comparison in the Static condition was significant $(t(21)=-2.7$, $p<.01)$ but the comparison in the Coordination condition was not significant at the adjusted alpha level $(\mathrm{t}(21)=-1.7, \mathrm{p}=.05)$.

In summary; we observed a clear egocentric effect for those in their 20 s in both feedback conditions, and for those in their 50s in the Static feedback condition. The group in their 60s showed no difference in coordination stability as a function of either the allocentric or egocentric constraint. 


\section{Discussion}

This exploratory report develops the hypothesis that the egocentric constraint on bimanual rhythmic movement coordination is about the haptic perception of phase, rather than muscle homology or neural encoding. We took bimanual coordination data from two existing studies and analysed coordination stability relative to the allocentric and egocentric definitions of relative phase. The goal was to identify any patterns in these data and to use these patterns to generate hypotheses that can be tested in more directed experiments.

Just to be clear: the reported analyses were not hypothesis driven, and the various post-hoc follow up tests we ran were explicitly chosen to examine the observed patterns in the data. These results should not be treated as anything other than some observed patterns until they are replicated and extended in hypothesis driven experiments.

With that in mind, we will now briefly summarise what we found and how the results relate to the various hypotheses about the egocentric constraint.

\section{Age Related Changes in the Egocentric Constraint}

These two studies (Ren et al, 2015; Zhu et al, 2017) are part of a larger programme investigating the source of a precipitous decline in learning rates that occurs in the 50s (Coats et al, 2012, 2014). Those studies focused on learning a $90^{\circ}$ coordination. Here, we focused on performance in the Baseline assessment session, in which participants were asked to move at $0^{\circ}$ or $180^{\circ}$ (allocentrically), anti-phase or in-phase (egocentrically).

In the combined Baseline data, we saw a clear egocentric effect in the 20s age group; in-phase movements were preferred over anti-phase movements. In the absence of allocentric direction information (with the Static feedback) this effect persisted in the 50s age group. But the 60s age group showed no preference; both movements were equally stable. Examination of the data in Figure 1 suggests that $0 \%$ Anti-Phase performance was fairly stable across the age groups, while the $180^{\circ} /$ In-Phase advantage decreased with age. 
The main novel result here is a change with age in the size of the egocentric effect on coordination stability.

\section{Explaining Age Related Changes in the Egocentric Constraint}

If the egocentric constraint is caused by muscle homology (implemented as interference during neural crosstalk) there is no obvious reason to expect age related changes in this interference. The cross-talk and higher-order muscle activation patterns are a function of the required coordinated movement, and the older participants were required to move at the same relative phases as the younger participants. The older adults did perform worse than the younger adults, raising the possibility that because their movements were less coordinated the cross-talk effect was reduced. But their performance was still fairly good (PTT20 around 0.6) which was comparable to the younger adults in the $0^{\circ} /$ Anti-Phase condition, and so they were presumably trying to produce coordinated neuromuscular activity. They simply gained no benefit from the egocentrically defined in-phase movement.

It's also not clear why there should be age-related changes to the neural coding of direction information from an egocentric to an allocentric frame of reference (Swinnen et al, 2002), at least in the absence of a change in the perception of that space. One reason might be that with age and greater experience, the allocentric coding is revealed to be more efficient or effective. But if this were the case, why would it take 60 years of experience to alter? And if the egocentric encoding was actually effective, why does it change in the 60s? While we agree with Swinnen et al that the way the brain interacts with space is obviously part of the story, without a focus on perception there is no clear mechanism for structuring that interaction.

We propose that the presence of age-related changes in the egocentric constraint on coordination stability could be caused by age-related perceptual changes in the haptic perception of relative phase. When we first identified the 50s Cliff (Coats et al, 2014) it was in the context of a unimanual coordination task in which people had to learn to move relative to a computer controlled dot in a 
coordination display. Those data were therefore structured only by the allocentric constraint, specifically the visual perception of relative phase (movements to produce $0^{\circ}$ were more stable than movements to produce $180^{\circ}$ in this study). We therefore proposed that a likely cause of the 50 s cliff was the well-known changes in the visual perception of relative motion information (e.g. Betts, Taylor, Sekuler \& Bennett, 2005; Snowden \& Kavenagh, 2006). We tested this hypothesis in the Ren et al and Zhu et al papers by comparing visual training to haptic training, and found the learning rate decline in both conditions. We concluded in Ren et al that this ruled out the visual motion perception explanation and pointed to a more general change in the perceptuo-motor process. However, on the basis of the Assessment data reported here, we now propose that we gave up on a perceptual explanation too quickly, and that these results warrant further investigation of the haptic, egocentric perception of relative phase.

\section{The Effect of Ageing on Haptic Perception}

While there are well documented age-related changes to the receptors of the haptic system (e.g. Corso, 1981), haptic perception of various properties (shape, curvature) via dynamic touch actually seem to remain intact across the lifespan (e.g. Carello, Thuot \& Turvey, 2000; Chang et al, 2008; Norman et al, 2010, 2013). Konczak et al (2012) did find age-related decrements in haptic discrimination of curvature.

The existing literature does not immediately support the existence of age related changes in haptic perception. We will note for now that existing work focuses on the perception of static spatial properties of objects, rather than the perception of dynamic, spatial-temporal, relative motion of the limbs. We know about decrements in the visual perception of this latter property due to work specifically testing that perception (e.g. Betts et al, 2005). In fact, the existence of the 50s Cliff in the haptic acquisition of a $90^{\circ}$ coordination may be interpreted as the first evidence for an age-related change in the haptic perception of information relevant to performance in this task. This remains a speculation, however, and requires careful hypothesis driven testing. In the next section we will 
review the tools and methods we have developed studying the allocentric perception of relative phase that can be applied here.

\section{Proposals for the Future}

Over the past 18 years or so, we have been investigating the visual, allocentric perception of relative phase, and that programme involved developing a variety of tools and analyses. We therefore propose pursuing the haptic, egocentric perception of relative phase using these tools.

\section{Judgment Studies}

The first set of studies establishing a role for perception involved judging mean relative phase and levels of phase variability in visual displays of computer generated movements (Bingham et al, 1999, 2000; Zaal et al, 2000). The critical pattern of results was that the coordination stability phenomena $\left(0^{\circ}\right.$ more stable than $180^{\circ}$, everything else unstable) persisted even in the absence of any limb movements, that is, motoric activity. Phase variability was clearly discriminated around $0^{\circ}$, less so around $180^{\circ}$ and not at all at $90^{\circ}$. This all supported the hypothesis that coordination stability is a function of perceptual stability.

We have already one haptic judgment study (Wilson, Bingham \& Craig, 2003) in which people tracked, rather than produced, coordinated rhythmic movements and then judged the variability in those movements. We replicated the visual judgment results and concluded the haptic perception of relative phase entailed using the same information variable as in vision. However, note that the movements were up-and-down finger movements, and so the allocentric and egocentric measurement of relative phase overlapped; the next step will be to replicate this study with side-toside, in-phase vs anti-phase movements to see if the haptic perception of coordination is in fact egocentric.

As part of the judgment studies, we also developed a method for selectively perturbing candidate visual information variables (Wilson \& Bingham, 2008). Perturbation methods are a tool of dynamical systems theory that have been used in studying movement dynamics (e.g. Kay, Kelso, 
Saltzman \& Schöner, 1987; Kay, Saltzman \& Kelso, 1991) and we adapted them to a perceptual psychophysics context. A perturbed information variable is still present in the display, but it is no longer informative about the underlying dynamics. If a person is using that variable to perceive those dynamics, breaking that link will break performance. We confirmed that the visual perception of relative phase generally ${ }^{1}$ entails the relative direction of motion, and also showed that people trained to perceive $90^{\circ}$ displays clearly had switched information variables, to relative position. All these methods can be readily adapted to the egocentric haptic domain.

\section{From Perception to Action}

We then moved to connect the perceptual results to the control of coordinated actions themselves. We have shown that manipulating the allocentric information for relative phase has predictable consequences for unimanual coordination stability (difficult coordinations such as $90^{\circ}$ movements become easy when the screen displays an easily discriminated coordinations such as $0^{\circ}$; Wilson et al, $2005 a, b)$. We then showed that perceptual learning of $90^{\circ}$ leads to improved coordination stability at $90^{\circ}$ without any practice of the movements; coordination stability is a function of perceptual stability (Wilson et al, 2010). Most recently, we have shown that the allocentric, visual information for relative phase supports transfer of learning of $90^{\circ}$ between the unimanual and bimanual versions of the task performed with coordination feedback (Snapp-Childs et al, 2015). In bimanual tasks, both visual and haptic information are available but people do not learn about haptically defined relative phase if the training used visual feedback (Zhu et al, 2017; Bingham et al, submitted), Learning does not transfer across visual and haptic modalities, supporting the different frames of reference analysis. Again, these methods are all readily adapted.

In summary, perceptual judgments, information perturbations, and training/transfer experiments are all tools that have revealed a great deal about the allocentric, visual perception of relative phase

\footnotetext{
${ }^{1}$ There is individual variation; some people use relative position at $0^{\circ}$ and $180^{\circ}$, and we are currently exploring this in more detail.
} 
but have yet to be deployed to the egocentric, haptic perception of relative phase. Part of the reason for this is that the egocentric constraints have long been framed in terms of muscle homology. Recognising that homology is not the only way to define an egocentric frame of reference (Swinnen et al, 2002) and engaging with the presence of an egocentric reference frame in haptic perceptual medium (Kappers, 2005, 2007; Kappers \& Bergmann Tiest, 2015; Turvey \& Fonseca, 2014) reframes the question to one in which perception could readily play a role and that this kind of research programme might be able to answer.

\section{Summary}

The stability of coordinated rhythmic movements are primarily determined by the target relative phase of the coordination. The evidence supports the idea that people measure relative phase in both possible frames of reference. The allocentric frame of reference comes from the visual perception of relative phase, defined in terms of the relative direction of motion and implemented in the external, allocentric optic array. The egocentric frame of reference has been proposed to come from neural crosstalk during the planning and production of actions, or from the neural encoding of the body's motions. The current exploratory analysis was designed to investigate a third explanation, that the egocentric frame comes from the haptic perception of relative phase, defined in terms of the relative direction of motion and implemented in the internal, egocentric haptic array. We analysed data from two existing studies (Ren et al, 2015; Zhu et al, 2017) and identified age related changes in the egocentric constraint on coordinated rhythmic movement stability. There is no obvious mechanism for age related changes in neural cross talk during or neural encoding of coordinated rhythmic movements, but perceptual changes are a common feature of ageing. Research on haptic perception and ageing do typically find that haptic perception does not get worse with age, but there is much more targeted work required before ageing effects can be ruled out. 
These data provide a foot in the door for a perceptual hypothesis, and should be used as preliminary results that can drive more specifically hypothesis driven work in the future using the tools developed to study the visual perception of relative phase. 


\section{References}

Amazeen, E., DaSilva, F., \& Amazeen, P. (2008). Visual-spatial and anatomical constraints interact in a bimanual coordination task with transformed visual feedback. Experimental Brain Research, 191(1), 13-24.

Beek, P. J., Peper, C. E., \& Daffertshofer, A. (2002). Modeling rhythmic interlimb coordination: Beyond the Haken-Kelso-Bunz model. Brain and Cognition, 48(1), 149-165.

Betts, L. R., Taylor, C. P., Sekuler, A.B., \& Bennett, P.J. (2005). Aging reduces center-surround antagonism in visual motion processing. Neuron 45, 361-366.

Bingham, G. P., Schmidt, R., \& Zaal, F. (1999). Visual perception of the relative phasing of human limb movements. Perception \& Psychophysics, 61(2), 246-258.

Bingham, G. P., Snapp-Childs, W., \& Zhu, Q. (submitted). Information about relative phase in bimanual coordination is modality specific (not amodal), but kinesthesis and vision can teach one another.

Bingham, G. P., Zaal, F. T., Shull, J. A., \& Collins, D. R. (2001). The effect of frequency on the visual perception of relative phase and phase variability of two oscillating objects. Experimental Brain Research, 136(4), 543-552.

Brandes, J., Rezvani, F., \& Heed, T. (2017). Abstract spatial, but not body-related, visual information guides bimanual coordination. Scientific Reports, 7(1), 16732.

Carello, C., Thuot, S., Turvey, M. T. (2000). Aging and the perception of a racquet's sweet spot. Human Movement Science, 19, 1-20.

Cattaert, D., Semjen, A., \& Summers, J. J. (1999). Simulating a neural cross-talk model for betweenhand interference during bimanual circle drawing. Biological Cybernetics, 81(4), 343-358. 
Chang, C. H., Wade, M. G., Stoffregen, T. A., \& Ho, H. Y. (2008). Length perception by dynamic touch: The effects of aging and experience. The Journals of Gerontology Series B: Psychological Sciences and Social Sciences, 63(3), 165-170.

Coats, R. O., Snapp-Childs, W., Wilson, A. D., \& Bingham, G. P. (2012). Perceptuo-motor learning rate declines by half from 20s to 70/80s. Experimental Brain Research, 225(1), 75-84.

Coates, R. O., Wilson, A. D., Snapp-Childs, W., Fath, A. J., \& Bingham, G. P. (2014). The 50s cliff: Perceptuo-motor learning rates across the lifespan. PLOSOne, 9(1), e85758

Daffertshofer, A., Peper, C. L. E., \& Beek, P. J. (2005). Stabilization of bimanual coordination due to active interhemispheric inhibition: a dynamical account. Biological Cybernetics, 92(2), 101109.

Georgopoulos, A. P. (1991). Higher order motor control. Annual Review of Neuroscience, 14, 361377.

Georgopoulos, A. P. (1995). Current issues in directional motor control. Trends in Neurosciences, 18, 506-510.

Haken, H., Kelso, J. S., \& Bunz, H. (1985). A theoretical model of phase transitions in human hand movements. Biological Cybernetics, 51(5), 347-356.

JASP Team (2018). JASP (Version 0.8.5) [Computer software].

Kelso, J. A. S. (1995). Dynamic Patterns. Cambridge, Mass.: MIT Press.

Kagerer, F. A., Summers, J. J., \& Semjen, A. (2003). Instabilities during antiphase bimanual movements: Are ipsilateral pathways involved? Experimental Brain Research, 151, 489-500.

Kappers, A. M. (2005, March). Intermediate frames of reference in haptically perceived parallelity. In Eurohaptics Conference, 2005 and Symposium on Haptic Interfaces for Virtual Environment and Teleoperator Systems, 2005. World Haptics 2005. First Joint (pp. 3-11). IEEE. 
Kappers, A. M. (2007). Haptic space processing--Allocentric and egocentric reference frames. Canadian Journal of Experimental Psychology/Revue canadienne de psychologie expérimentale, 61(3), 208.

Kappers, A. M. L. \& Bergmann Tiest, W. M. (2015) Haptic Perception. In The Cambridge Handbook of Applied Perception Research. Hoffman, R. R., Scerbo, M. W., Parasuraman, R. \& Szalma, J. L. (eds.). New York, NY: Cambridge University Press, p. 345-365.

Kay, B. A., Kelso, J. A. S., Saltzman, E. L., \& Schöner. G. (1987).Space-time behavior of single and bimanual rhythmical movements: Data and limit cycle model. Journal of Experimental Psychology: Human Perception and Performance, 13, 178-192.

Kay, B. A., Saltzman, E. L., \& Kelso, J. A. S. (1991). Steady-state and perturbed rhythmical movements: A dynamical analysis. Journal of Experimental Psychology: Human Perception and Performance, 17, 183-197.

Konczak, J., Sciutti, A., Avanzino, L., Squeri, V., Gori, M., Masia, L., Abbruzzese, G., \& Sandini, G. (2012). Parkinson's disease accelerates age-related decline in haptic perception by altering somatosensory integration. Brain, 135(11), 3371-3379.

Li, Y., Levin, O., Forner-Cordero, A., Ronsse, R., \& Swinnen, S. P. (2009). Coordination of complex bimanual multijoint movements under increasing cycling frequencies: the prevalence of mirror-image and translational symmetry. Acta Psychologica, 130(3), 183-195.

Marteniuk, R. G., MacKenzie, C. L., \& Baba, D. M. (1984). Bimanual movement control: Information processing and interaction effects. The Quarterly Journal of Experimental Psychology Section A, 36(2), 335-365.

Mechsner, F., Kerzel, D., Knoblich, G., \& Prinz, W. (2001). Perceptual basis of bimanual coordination. Nature, 414(6859), 69. 
Norman, J. F., Kappers, A. M., Beers, A. M., Scott, A. K., Norman, H. F., \& Koenderink, J. J. (2011). Aging and the haptic perception of 3D surface shape. Attention, Perception, \& Psychophysics, 73(3), 908-918.

Norman, J. F., Crabtree, C. E., Norman, H. F., Moncrief, B. K., Herrmann, M., \& Kapley, N. (2006). Aging and the visual, haptic, and cross-modal perception of natural object shape. Perception, 35(10), 1383-1395.

Peper, C. E., de Boer, B. J., de Poel, H. J., \& Beek, P. J. (2008). Interlimb coupling strength scales with movement amplitude. Neuroscience Letters, 437, 10-14.

Pickavance, J., Azmoodeh, A., \& Wilson, A. D. (2018). Pickavance et al 2018 Data and paper. Retrieved from osf.io/z7c9q

Ren, J., Huang, S., Zhang, J., Zhu, Q., Wilson, A. D., Snapp-Childs, W. \& Bingham, G. P. (2015). The 50s Cliff: A Decline in perceptuo-motor learning, not a deficit in visual motion perception. PLoS ONE 10(4): e0121708.

Snapp-Childs, W., Wilson, A. D., \& Bingham, G. P. (2011). The stability of rhythmic movement coordination depends on relative speed: the Bingham model supported. Experimental Brain Research, 215(2), 89.

Snapp-Childs, W., Wilson, A. D. \& Bingham, G. P. (2015). Transfer of learning between unimanual and bimanual rhythmic movement coordination: Transfer is a function of the task dynamic. Experimental Brain Research 233(7), 2225-38.

Snowden RJ, Kavanagh E (2006) Motion perception in the ageing visual system: Minimum motion, motion coherence, and speed discrimination thresholds. Perception 35, 9-24.

Swinnen, S. P. (2002). Intermanual coordination: from behavioural principles to neural-network interactions. Nature Reviews: Neuroscience, 3(5), 348-359. 
Swinnen, S. P., Dounskaia, N., \& Duysens, J. (2002). Patterns of bimanual interference reveal movement encoding within a radial egocentric reference frame. Journal of Cognitive Neuroscience, 14(3), 463-471.

Swinnen, S. P., Dounskaia, N., Levin, O., \& Duysens, J. (2001). Constraints during bimanual coordination: the role of direction in relation to amplitude and force requirements. Behavioural Brain Research, 123(2), 201-218.

Swinnen, S. P., Young, D. E., Walter, C. B., \& Serrien, D. J. (1991). Control of asymmetrical bimanual movements. Experimental Brain Research, 85(1), 163-173.

Temprado, J., Swinnen, S., Carson, R., Tourment, A., \& Laurent, M. (2003). Interaction of directional, neuromuscular and egocentric constraints on the stability of preferred bimanual coordination patterns. Human Movement Science, 22(3), 339-363.

Turvey, M. T., \& Fonseca, S. T. (2014). The medium of haptic perception: a tensegrity hypothesis. Journal of Motor Behavior, 46(3), 143-187.

Wilson, A. D., \& Bingham, G. P. (2008). Identifying the information for the visual perception of relative phase. Perception \& Psychophysics, 70(3), 465-476.

Wilson, A. D., Bingham, G. P., \& Craig, J. C. (2003). Proprioceptive perception of phase variability. Journal of Experimental Psychology: Human Perception and Performance, 29(6), 1179.

Wilson, A. D., Snapp-Childs, W., \& Bingham, G. P. (2010). Perceptual learning immediately yields new stable motor coordination. Journal of Experimental Psychology: Human Perception and Performance, 36(6), 1508.

Wilson, A. D., Snapp-Childs, W., Coats, R., \& Bingham, G. P. (2010). Learning a coordinated rhythmic movement with task-appropriate coordination feedback. Experimental Brain Research, 205(4), 513-520. 
Wilson, A. D., Collins, D. R., \& Bingham, G. P. (2005a). Perceptual coupling in rhythmic movement coordination: stable perception leads to stable action. Experimental Brain Research, 164(4), 517-528.

Wilson, A. D., Collins, D. R., \& Bingham, G. P. (2005b). Human movement coordination implicates relative direction as the information for relative phase. Experimental Brain Research, 165(3), 351-361.

Zhu, Q., Mirich, T., Huang, S., Snapp-Childs, W., \& Bingham, G. P. (2017). When kinesthetic information is neglected in learning a novel bimanual rhythmic coordination. Attention, Perception, \& Psychophysics, 79(6), 1830-1840. 
Figures

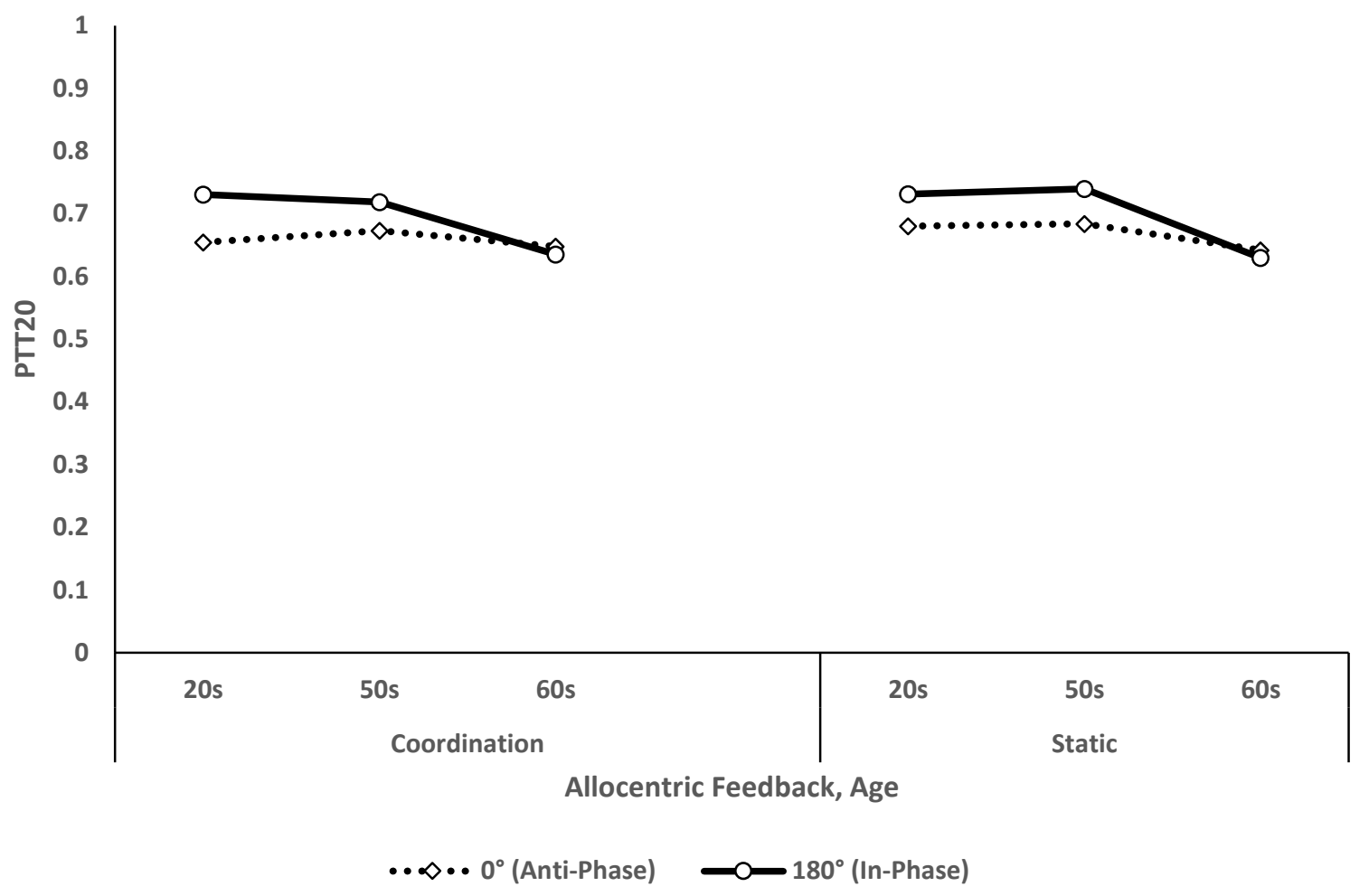

Figure 1.

Combined Baseline assessment data from Ren et al (2015) and Zhu et al (2017). 